\title{
Comparative Study of Channel Coding Schemes for 5G
}

Walled Abdulwhab, Abdulkareem Kadhim

Al-Nahrain University

Iraq

walled.khalid@coie-nahrain.edu.iq

abdulkareem.a@coie.nahrainuniv.edu.iq

ABSTRACT: Channel coding is required to correct transmission errors that are caused by noise, interference and poor signal strength. $3 G$ and $4 G$ systems already used turbo code for error correction. $3 G P P$ and other standardization bodies are currently debating whether to use Low Density Parity Check (LDPC) or polar code instead in 5G. In this paper we look into $5 G$ requirements for channel coding and review candidate channel coding schemes for $5 G$. A comparative study is presented for possible channel coding candidates of $5 G$ covering Convolutional, Turbo, LDPC, and Polar codes. It seems that polar code with Successive Cancellation List (SCL) decoding using small list length (such as 8) is a promising choice for short message lengths ( $\leq 128$ bits) due to its error performance and relatively low complexity. Adopting non-binary LDPC can provide good performance on the expense of increased complexity but with better spectral efficiency. Considering the implementation, polar code with decoding algorithms based on SCL required small area and low power consumption when compared to LDPC codes. For larger message lengths ( $\geq 256$ bits) turbo code can provide better performance at low coding rates $(<1 / 2)$.

Keywords: 5G, Channel Coding, Convolutional Code, Turbo Code, LDPC, Polar Code

DOI: $10.6025 / \mathrm{jes} / 2018 / 8 / 3 / 95-102$

Received: 3 April 2018, Revised 12 May 2018, Accepted 19 May 2018

(C) 2018 DLINE. All Rights Reserved

\section{Introduction}

In G1 mobile system the focus was on providing a voice service, while in G2 the trend is focused on enhancement of the voice service and providing text message service. The $3 \mathrm{G}$ mobile system integrated all the services provided by $2 \mathrm{G}$ with mobile Internet services [1-4]. 4G demanded more in developing the previous generation services by supporting high quality multimedia for mobile nodes. Recently, $5 \mathrm{G}$ is aiming to support new types of mobile services with improved features and performance regarding transmission quality and resources [1,3-6]. The key issue in providing better quality is to use error correction coding to improve the error performance of the recovered data at the receiving end. Advanced channel coding schemes are required to achieve error free transmission for modern systems highlighted by more users, better transmission quality, high bandwidth efficiency, low latency, and less consumed energy $[1,7,8]$.

Journal of Electronic Systems Volume 8 Number 3 September 2018 
The early works on error correction codes produced useful codes with good performance. Unfortunately, these codes did not achieve or even approached the channel capacity limit set forth by Shannon [9,10 ]. Reed-Solomon (RS) and Bose-ChaudhuriHocquenghem $(\mathrm{BCH})$ codes are good examples of such codes and are still being used widely. The discovery of turbo codes in the 1990s showed the first channel coding method that approaching the Shannon limit. Low-Density Parity Check (LDPC) code is discovered in the 1960's by Robert Gallager before turbo code and it is also a capacity approaching code. It is only become useful code when David McKay in 1997 discovered a simple decoding algorithm [11,12]. Turbo or a LDPC code are already used in diverse of applications such as video broadcasting, wireless and wired communications, and data storage. The latest findings on the road to achieving channel capacity are polar codes. Polar codes, invented by Arýkan in 2009 [11,13,14], have a nonrandom construction making the implementation of their encoders and decoders simpler than those of LDPC or turbo codes. Channel polarization is used in polar code to achieve the symmetric capacity of memoryless channel as their length tends to infinity $[11,14]$.

Many works in the literatures introduced comparative studies among different error correction schemes that may serve as possible candidates for $5 \mathrm{G}[1,5,6,7,11,13,15]$. Different environments and code parameters are considered in these references. Convolution, Turbo, LDPC, and Polar codes are the possible candidates. Unfortunately, there is no work that unifies the mentioned codes in fair comparison. In this paper the results of different works are reviewed and summarized to cover different coding schemes with wide range of parameters and environments to make more realistic conclusion regarding the most promising coding schemes for the $5 \mathrm{G}$.

The remaining sections of this paper are organized as follows; in Section 2 we present the requirements for $5 \mathrm{G}$. Useful tabulated error performance results together with complexity and other important coding features are discussed in Section 3 . The assessment of the results is covered in Section 4. Finally, the main conclusion remarks are presented in Section 5.

\section{Requirements for $5 \mathrm{~g}$}

It is important to look at the main requirements of $5 \mathrm{G}$ that are set forth by standardization bodies and vendors. According to such efforts, the requirements can be classified into network requirements and wireless transmission requirements [8, 16-18]. The main wireless transmission requirements which are related to the channel coding issues are; High Throughput, Low Latency, Low Error Rate, High Spectral Efficiency, Flexibility, and Energy Efficiency.

\section{Review of useful Results}

In this section the available results of different works related to channel coding for $5 \mathrm{G}$ systems are summarized. Although some of test conditions related to code parameters and environments are not the same, we tried here to unify the presented efforts in a way to make clear picture of all channel coding in unified manner so that a fair comparison can be made. The results are arranged according to given channel coding scheme in tables.

The performance of LTE turbo code is presented in TABLE I in terms of BER and Block Error Rate (BLER) with varying length and different coding rates (from $1 / 3$ to 5/6) [5,11,15]. BPSK and 16-QAM signals operating over both AWGN and fading wireless channels are considered. The signal to noise power ratio is given by $E_{b} / N_{0}$ (in $\mathrm{dB}$ ) are shown with the corresponding level of error rates (BER and BLER). The values of such error rates considered for comparison here is around $10^{-4}$ or less, depending on the available results and test conditions. The required error rate for $5 \mathrm{G}$ is $10^{-5}[1,7]$. As a general remark for Table 1 , the best results are obtained for code lengths of 1024 or larger $[5,11,15]$. This is achieved on the expense of increased complexity for both AWGN and fading wireless channels using 8 iterations or more in decoding $[5,11,15]$. The complexity is linearly related to the information block length as for the case when the length is changed from 128 to 512 [5] or from 128 to 4096 [1,15]. In [6], further results (not shown in Table 1) for Duo-Binary turbo code with different modulation levels and different coding rates using 10 iterations in decoding and fixed block length of 8000 are presented. Spectral efficiencies ranging from 1 to $5 \mathrm{bps} / \mathrm{Hz}$ at acceptable $E_{b} / N_{0}$ over AWGN with BLER of $10^{-4}$ are demonstrated [6]. Flexibility in selecting modulation levels and coding rates supported 188 different message lengths [7]. It can achieve the flexibility requirement for 5G with attractive energy and area efficiency, but this is not the case for very high throughput [1]. Thus providing back-word compatibility with previous mobile generations while maintaining reduced implementation complexity [7]. For given structure the energy and area efficiency is not changed with coding rate. Turbo code suffer from poor performance at rate higher than $1 / 3$ due to error floor caused by puncturing [1].

The result of convolutional code that is being used in LTE is shown in Table 2 [5,11]. Two Tail-Biting Convolutional Codes J6 Journal of Electronic Systems Volume 8 Number 3 September 2018 
(TBCC1 and TBCC2) are considered here [5]. For lengths greater than or equal to 512, the performance did not change for the same BER (around $2 \times 10^{-4}$ in Table 2) with better performance for lower coding rates as expected [11]. In addition, the performance is improved as the number of memory elements is increased ( 6 for TBCC1 and 10 for TBCC2). This is obtained at the cost of increasing complexity and hence increased power consumption which is a critical factor in 5G. On the other hand, for large memory convolutional code, the added complexity due to larger code length is relatively small where the complexity is increased by factor in the rang 1.5 to 1.8 for smaller memory and by factor in the range of 1.3 to 1.6 for larger memory length at each time the length is duplicated [5].

\begin{tabular}{|c|c|c|c|c|c|c|c|c|}
\hline \multirow{3}{*}{$\begin{array}{l}\text { Block } \\
\text { Length }\end{array}$} & \multirow{3}{*}{$\begin{array}{l}\text { Refer- } \\
\text { ences }\end{array}$} & \multirow{3}{*}{ Parameters } & \multirow{2}{*}{\multicolumn{3}{|c|}{$\frac{\mathrm{E}_{\mathrm{b}} / \mathrm{N}_{\mathrm{o}} \text { in } \mathrm{dB} \text { (BER) }}{\text { Coding Rate }}$}} & \multicolumn{3}{|c|}{$E_{b} / N_{o}$ in dB ( BLER) } \\
\hline & & & & & & & oding Rat & \\
\hline & & & $1 / 3$ & $1 / 2$ & $2 / 3$ & $1 / 3$ & $1 / 2$ & $2 / 3$ \\
\hline \multirow{2}{*}{128} & \multirow{2}{*}[4,14]{} & AWGNQAM & $3.5\left(10^{-4}\right)$ & $3.8\left(10^{-4}\right)$ & $4.4\left(10^{-4}\right)$ & $4\left(2 \times 10^{-4}\right)$ & $4\left(4 \times 10^{-4}\right)$ & $5\left(10^{-4}\right)$ \\
\hline & & FadingQAM & $4.7 \quad\left(10^{-4}\right)$ & $5\left(10^{-4}\right)$ & $5\left(10^{-3}\right)$ & $5\left(5 \times 10^{-4}\right)$ & $5\left(2 \times 10^{-3}\right)$ & $5\left(1.5 \times 10^{-2}\right)$ \\
\hline 256 & {$[4]$} & AWGN BPSK, $\mathrm{I}=8$ & $0.3\left(10^{-5}\right)$ & $2.8\left(10^{-5}\right)$ & $5\left(10^{-5}\right)$ & ------- & $3.5\left(10^{-5}\right)$ & ------- \\
\hline \multirow{3}{*}{512} & \multirow{3}{*}[4,14]{} & AWGNQAM & $1.9\left(10^{-4}\right)$ & $2.3\left(10^{-4}\right)$ & $3\left(10^{-4}\right)$ & $2.4\left(10^{-4}\right)$ & $2.6\left(10^{-4}\right)$ & $3.7\left(10^{-4}\right)$ \\
\hline & & FadingQAM & $2.5\left(10^{-4}\right)$ & $3.2\left(10^{-4}\right)$ & $4.5\left(10^{-4}\right)$ & $3.5\left(10^{-4}\right)$ & $4.3\left(10^{-4}\right)$ & $5\left(2 \times 10^{-3}\right)$ \\
\hline & & AWGN BPSK, $\mathrm{I}=8$ & $-0.1\left(10^{-5}\right)$ & $2.2\left(10^{-5}\right)$ & $4.3\left(10^{-5}\right)$ & --- & $2.5\left(10^{-5}\right)$ & ---- \\
\hline \multirow{3}{*}{1024} & \multirow{3}{*}[10,14]{} & AWGNQAM & $1.5\left(10^{-4}\right)$ & $1.7\left(10^{-4}\right)$ & $2.7\left(10^{-4}\right)$ & $1.9\left(10^{-4}\right)$ & $2.2\left(10^{-4}\right)$ & $3\left(10^{-4}\right)$ \\
\hline & & FadingQAM & $2\left(10^{-4}\right)$ & $2.5\left(10^{-4}\right)$ & $3.8\left(10^{-4}\right)$ & $3\left(10^{-4}\right)$ & $3.5\left(10^{-4}\right)$ & $5\left(10^{-4}\right)$ \\
\hline & & AWGN BPSK, I=8 & $-0.5\left(10^{-5}\right)$ & $2\left(10^{-5}\right)$ & $4.1\left(10^{-5}\right)$ & ----- & ----- & -ב- \\
\hline \multirow{2}{*}{2048} & \multirow{2}{*}[14]{} & AWGNQAM & $1.1\left(10^{-4}\right)$ & $1.5\left(10^{-4}\right)$ & $2.4\left(10^{-4}\right)$ & $1.5\left(10^{-4}\right)$ & $2\left(10^{-4}\right)$ & $3.2 \quad\left(10^{-4}\right)$ \\
\hline & & AWGN BPSK, $\mathrm{I}=8$ & $-0.7\left(10^{-5}\right)$ & $1.7\left(10^{-5}\right)$ & $3.8\left(10^{-5}\right)$ & --- & -ב--- & -----' \\
\hline
\end{tabular}

Table 1. Performance of Turbo Code with different parameters and environments $a-E_{b} /$ No in $\mathrm{dB}$ and corresponding BER for different coding rates of LTE convolutional encoder [10].

\begin{tabular}{|l|l|l|l|c|}
\hline \multirow{2}{*}{ BlockLength } & \multicolumn{4}{|c|}{ Encoder Rate } \\
\cline { 2 - 5 } & \multicolumn{1}{|c|}{$\mathbf{1 / 3}$} & $\mathbf{1 / 2}$ & $\mathbf{2 / 3}$ & $\mathbf{5 / 6}$ \\
\hline 256 & $2\left(10^{-5}\right)$ & $4\left(10^{-5}\right)$ & $5.7\left(10^{-5}\right)$ & $8\left(10^{-5}\right)$ \\
\hline 512 & $1\left(3 \times 10^{-4}\right)$ & $3\left(4 \times 10^{-4}\right) 5$ & $\left(2 \times 10^{-4}\right)$ & $8\left(10^{-5}\right)$ \\
\hline 1024 & $1\left(2 \times 10^{-4}\right)$ & $3\left(4 \times 10^{-4}\right) 5$ & $\left(2 \times 10^{-4}\right)$ & $8\left(10^{-5}\right)$ \\
\hline 2048 & $1\left(3 \times 10^{-4}\right)$ & $3\left(4 \times 10^{-4}\right)$ & $5\left(2 \times 10^{-4}\right)$ & $8\left(10^{-5}\right)$ \\
\hline
\end{tabular}

$b-E_{b} /$ No in $\mathrm{dB}$ and corresponding BLER for two tail bit convolutional encoders with coding rate of $1 / 2[4]$. 


\begin{tabular}{|l|l|l|c|}
\hline Block Length & $\mathbf{1 2 8}$ & $\mathbf{2 5 6}$ & $\mathbf{5 1 2}$ \\
\hline TBCC1 & $4.5\left(10^{-4}\right)$ & $4.5\left(2 \times 10^{-4}\right)$ & $4\left(2 \times 10^{-3}\right)$ \\
\hline TBCC2 & $3.9\left(10^{-5}\right)$ & $4\left(2 \times 10^{-5}\right)$ & $4\left(3 \times 10^{-5}\right)$ \\
\hline
\end{tabular}

Table 2. Performance of convolutional code using BPSK

LDPC codes are widely implemented in hardware commercially and supporting throughputs of several Gbps with attractive energy and area efficiency [1]. Table 3 shows the performance of LDPC. As the code length is increased both BER and BLER are improved in terms of $E_{b} / N_{0}$ for different modulation levels $[5,11,13,15]$. For BPSK the performance is improved as the coding rate is increased for large of decoding iterations $(\geq 10)$ especially with large coding rate [13]. The complexity is increased by a factor of 2 as the coding length is doubled [5]. Also the complexity is increased by a factor of 32 (at rates 1/3 and 2/3) and factor of 34 (at rate 1/2) as the length is increased from 128 to 4069 with Quasi Cyclic LDPC (QC-LDPC) and offset min- sum decoder [15]. As shown in Table 3, for Non-Binary LDPC (NB-LDPC) using GF (256), the performance is improved as the code length is increased for coding rate of $1 / 2$ but at the cost of increased complexity compared to Binary LDPC (B-LDPC) by a factor of 40 for the same coding length and rate [5]. Regular $(2,4)$ NB-LDPC, whose results are not shown in Table 3, with different QAM modulation levels, can achieve spectral efficiencies up to $5.5 \mathrm{bps} / \mathrm{Hz}$ over acceptable $E_{b} / N_{0}$ range [19]. This is obtained with block lengths of 2000 and 8000 over AWGN resulting in BLER of about $10^{-5}$ [6]. Further, LDPC that is used in WiMax can support 76 different combinations of coding lengths and rates, while LDPC which is used in WiFi can support 12 different combinations of coding lengths and rates [20 ]. The flexibility of LDPC code is far below the requirements of $5 \mathrm{G}$ services. The complexity of LDPC decoders become larger when the flexibility is increased [1]. For all schemes of LDPC tested, there is no back-word compatibility with previous mobile generations [7]. Although the decoders of LDPC have large degree of parallelism, this parallelism may be not achieved at all block lengths (poor performance for short block lengths). The area efficiency is reduced as the rate is decreased [1].

The performance of polar code is shown in Table 4. The error performance of such code is improved as the code length is increased at all coding rates, modulation levels, list size and partition considered in decoding [5,11-15]. The performance of polar code is also improved as the list length $(L)$ increased at the cost of increased decoding complexity [1]. The factor of increased complexity is about 50 as the list size is increased from 32 to 1024 [5]. In addition the complexity is increased by factor of about 10 times when using list of size 8 as compared to SC decoders of code lengths 128 and 4096 with different coding rates [15]. The improvement in performance is reduced in Partitioned Successive Cancellation List (PSCL) decoding compared to Successive Cancelation List (SCL) decoding algorithms [13]. PSCL achieved lower complexity in terms of area and power consumption compared to SCL and Fast SSCL using $L=2, C R C=8, R=1 / 2$ and partition $=2$ [13]. The complexity of SCL depends mainly on the list size, number of partitions and code length but not on coding rate [1,15]. A list length of 4 is widely used while that of 8 could be used with short block lengths only [1]. Also, the area efficiency of the code is decreased for short block length especially at low coding rate [1]. As the flexibility of polar code is concerned, a limited number of combinations for coding lengths and rates are supported without back-word compatibility with previous mobile generations [7]. There is no commercial implementation ready for polar codes at present time rising suspicious for the availability and maturity of decoder hardware [1].

\section{Assessment of Results}

At short lengths of 128 or below the performance of polar code with SCL decoding having different list lengths $(L=8,32$, and 1024) outperforms LDPC, Turbo, and convolutional codes over the range of coding rates considered especially at low coding rates. Turbo code complexity is greater than that of polar codes with SCL having list length and CRC of 8 , by factors ranging from 6 to 12 for low to high rates, respectively. The corresponding factors for LDPC are about 4.4 to 6 for high to low rates, respectively [15]. At coding length of 256 the performance of turbo code is better than that of LDPC, polar with SCL ( $L=4$ and 8 ), and convolutional code at low coding rates. The performance shows about $1 \mathrm{~dB}$ advantage in turbo code over polar code with SCL $(\mathrm{L}=8)$ at the cost of increased complexity. At higher coding rates LDPC shows the best error performance, while polar code with SCL $(L=32$ and 1024) or NB-LDPC outperform turbo code at coding rate of $1 / 2$ by about $1 \mathrm{~dB}$ at the cost of increased complexity [5].

At coding length of 512, the performance of both turbo and LDPC codes are almost identical with little advantage of turbo at low 98 Journal of Electronic Systems Volume 8 Number 3 September 2018 


\begin{tabular}{|c|c|c|c|c|c|c|c|c|c|}
\hline \multirow{3}{*}{$\begin{array}{l}\text { Block } \\
\text { Length }\end{array}$} & \multirow{3}{*}{$\begin{array}{l}\text { Refer } \\
\text { ences }\end{array}$} & \multirow{3}{*}{ Parameters } & \multicolumn{4}{|c|}{$E_{b} / N_{0}$ in dB (BER) } & \multirow{2}{*}{\multicolumn{3}{|c|}{$\begin{array}{c}\mathrm{E}_{\mathrm{b}} / \mathrm{N}_{\mathrm{o}} \text { in } \mathrm{dB} \text { (BLER) } \\
\text { Coding Rate }\end{array}$}} \\
\hline & & & & Coding 1 & Rate & & & & \\
\hline & & & $1 / 3$ & $1 / 2$ & $2 / 3$ & $5 / 6$ & $1 / 3$ & $1 / 2$ & $2 / 3$ \\
\hline \multirow{4}{*}{128} & {$[14]$} & AWGN, QAM, QC, I=50 & $3.8\left(10^{-4}\right)$ & $4\left(10^{-4}\right)$ & $4\left(10^{-4}\right)$ & ----- & $4\left(10^{-3}\right)$ & $4\left(6 \times 10^{-4}\right)$ & $4\left(3 \times 10^{-3}\right)$ \\
\hline & [14] & $\begin{array}{l}\text { Fading, QAM, } \\
\text { QC-LDPC, I=50 }\end{array}$ & $5\left(10^{-4}\right)$ & $5\left(10^{-4}\right)$ & $5\left(10^{-4}\right)$ & ----- & $5\left(1.4 \times 10^{-3}\right)$ & $5\left(1.7 \times 10^{-3}\right)$ & $5\left(10^{-2}\right)$ \\
\hline & {$[4]$} & AWGN, BPSK, AR3A & ----- & ------ & ----- & ----- & ---- & $3.9\left(10^{-5}\right)$ & ------ \\
\hline & [4] & AWGN, BPSK,NB-LDPC & ----- & ----- & ----- & ------ & ----- & $3.4\left(10^{-5}\right)$ & ----- \\
\hline \multirow{3}{*}{256} & [10] & AWGN, BPSK, $\mathrm{I}=16$ & $1\left(10^{-5}\right)$ & $3.2\left(10^{-5}\right)$ & $5.4\left(10^{-5}\right)$ & $7.3\left(10^{-5}\right)$ & ----- & ----- & ----- \\
\hline & {$[4]$} & AWGN, BPSK, AR3A & ----- & ----- & ----- & ----- & - & $3.6\left(10^{-5}\right)$ & ----- \\
\hline & [4] & $\begin{array}{l}\text { AWGN, BPSK, } \\
\text { NB GF(256) }\end{array}$ & ----- & ---- & ----- & ----- & ----- & $2.5\left(10^{-5}\right)$ & ----- \\
\hline \multirow{8}{*}{512} & [14] & AWGN, QAM,QC, I=50 & $2.2\left(10^{4}\right)$ & $2.3\left(10^{-4}\right)$ & $3\left(10^{-4}\right)$ & - ---- & $2.4\left(10^{-4}\right)$ & $2.7\left(10^{4}\right)$ & $3.5\left(10^{4}\right)$ \\
\hline & [14] & Fading, QAM,QC, I=50 & $2.8\left(10^{-4}\right)$ & $3.2\left(10^{-4}\right)$ & $4.2\left(10^{-4}\right)$ & ----- & $3\left(3 \times 10^{-4}\right)$ & $4\left(10^{-4}\right)$ & $5\left(10^{-4}\right)$ \\
\hline & [10] & $\begin{array}{l}\text { AWGN, BPSK, 802.16, } \\
\mathrm{I}=16\end{array}$ & $0.5\left(10^{-5}\right)$ & $2.6\left(10^{-5}\right)$ & $4.7\left(10^{-5}\right)$ & $6.7\left(10^{-5}\right)$ & - & ----- & ----- \\
\hline & [4] & AWGN, BPSK, AR3A & ----- & ----- & ----- & ----- & ----- & $2.7\left(6 \times 10^{-5}\right)$ & ----- \\
\hline & [4] & AWGN, BPSK,NB-LDPC & ----- & ----- & ----- & ----- & ----- & $2.1\left(10^{-5}\right)$ & ----- \\
\hline & [12] & $\begin{array}{l}\text { AWGN, BPSK, 802.16, } \\
\mathrm{I}=5\end{array}$ & ----- & ----- & ----- & ----- & ----- & $4.5\left(10^{-4}\right)$ & $4.5\left(10^{-5}\right)$ \\
\hline & [12] & $\begin{array}{l}\text { AWGN, BPSK, 802.16, } \\
\mathrm{I}=10\end{array}$ & ---- & ----- & ----- & ----- & ---- & $4.7\left(10^{-5}\right)$ & $4.1\left(10^{-5}\right)$ \\
\hline & [12] & $\begin{array}{l}\text { AWGN, BPSK, 802.16, } \\
\mathrm{I}=20\end{array}$ & ----- & ---- & ----- & ----- & - & $4.7\left(10^{-5}\right)$ & $4.1\left(10^{-5}\right)$ \\
\hline \multirow{3}{*}{1024} & [14] & AWGN, QAM,QC, I=50 & $1.7\left(10^{4}\right)$ & $2\left(10^{-4}\right)$ & $2.6\left(10^{-4}\right)$ & ----- & $2\left(10^{-3}\right)$ & $2\left(4 \times 10^{-4}\right)$ & $2.8\left(10^{-4}\right)$ \\
\hline & {$[14]$} & Fading, QAM,QC, I=50 & $2.2\left(10^{-4}\right)$ & $2.5\left(10^{-4}\right)$ & $3.5\left(10^{-4}\right)$ & ----- & $2.4\left(10^{-4}\right)$ & $3\left(10^{-4}\right)$ & $4\left(7 \times 10^{-4}\right)$ \\
\hline & 10 & $\begin{array}{l}\text { AWGN, BPSK,I=16, } \\
802.16\end{array}$ & $0.1\left(10^{-5}\right)$ & $2.4\left(10^{-5}\right)$ & $4.3\left(10^{-5}\right)$ & $6.4\left(10^{-5}\right)$ & ----- & ----- & ----- \\
\hline \multirow{2}{*}{2048} & {$[14]$} & AWGN, QAM,QC, I=50 & $1\left(6 \times 10^{-3}\right)$ & $1.7\left(10^{-4}\right)$ & $2.3\left(10^{-4}\right)$ & ---- & $1\left(7 \times 10^{-2}\right)$ & $2\left(10^{-4}\right)$ & $2.5\left(10^{-4}\right)$ \\
\hline & 10 & AWGN, BPSK, I=16 & $-0.2\left(10^{-5}\right)$ & $2.1\left(10^{-5}\right)$ & $4\left(10^{-5}\right)$ & $6.1\left(10^{-5}\right)$ & -ב--- & ---- & ----- \\
\hline
\end{tabular}

Table 3. Performance of LDPC code for different coding rates and environments $(\mathrm{QC}=$ Quasi Cyclic, $\mathrm{QAM}=16 \mathrm{QAM}, \mathrm{NB}=$ Nonbinary, $\mathrm{AR} 3 \mathrm{~A}=$ Accumulate - Repeat - Accumulate $)$ 


\begin{tabular}{|c|c|c|c|c|c|c|c|c|c|c|}
\hline \multirow{3}{*}{$\begin{array}{l}\text { Block } \\
\text { Length }\end{array}$} & \multirow{3}{*}{ References } & \multirow{3}{*}{ Parameters } & \multicolumn{4}{|c|}{$E_{b} / N_{0}$ in dB (BER) } & \multicolumn{4}{|c|}{$E_{b} / N_{o}$ in dB (BLER) } \\
\hline & & & \multicolumn{4}{|c|}{ Coding Rate } & \multicolumn{4}{|c|}{ Coding Rate } \\
\hline & & & $1 / 3$ & $1 / 2$ & $2 / 3$ & $5 / 6$ & $1 / 6$ & $1 / 3$ & $1 / 2$ & $2 / 3$ \\
\hline \multirow{4}{*}{128} & [14] & $\begin{array}{l}\text { AWGN, QAM, } \\
\mathrm{L}=8, \mathrm{CRC}=8\end{array}$ & $2.8\left(10^{-4}\right)$ & $3.3\left(10^{-4}\right)$ & $3.7\left(10^{-4}\right)$ & ---- & ------ & $3\left(10^{-4}\right)$ & $3.8\left(10^{-4}\right)$ & $4\left(10^{-4}\right)$ \\
\hline & {$[14]$} & $\begin{array}{l}\text { Fading, QAM, } \\
\mathrm{L}=8, \mathrm{CRC}=8\end{array}$ & $4.2\left(10^{-4}\right)$ & $4.8\left(10^{-4}\right)$ & $5\left(8 \times 10^{-4}\right)$ & $\mid----$ & ----- & $4.7\left(10^{-4}\right)$ & $5\left(4 \times 10^{-4}\right)$ & $5\left(10^{-2}\right)$ \\
\hline & {$[4]$} & $\begin{array}{l}\text { AWGN, BPSK, } \\
\mathrm{L}=32, \mathrm{CRC}=16\end{array}$ & ----- & ----- & ----- & |---- & ----- & ------ & $3.5\left(10^{-5}\right)$ & ----- \\
\hline & {$[4]$} & $\begin{array}{l}\text { AWG, BPSK, } \\
L=1024, C R C=16\end{array}$ & ----- & ----- & ----- & |---- & ----- & ---- & $3\left(10^{-5}\right)$ & ----- \\
\hline \multirow{4}{*}{256} & {$[10,12]$} & $\begin{array}{l}\text { AWGN, BPSK, } \\
\mathrm{L}=4, \mathrm{CRC}=8\end{array}$ & $1.3\left(10^{-5}\right)$ & $3.5\left(10^{-5}\right)$ & $5.9\left(10^{-5}\right)$ & $\begin{array}{l}8.3 \\
\left(10^{-5}\right)\end{array}$ & $\begin{array}{l}4.4 \\
\left(10^{-4}\right)\end{array}$ & $3.3\left(10^{-4}\right)$ & $3.9\left(10^{-5}\right)$ & $4.2\left(10^{-4}\right)$ \\
\hline & {$[12]$} & $\begin{array}{l}\text { AWGN, BPSK, } \\
\mathrm{L}=8, \mathrm{CRC}=8\end{array}$ & ----- & ----- & ----- & |---- & $\begin{array}{l}4.2 \\
\left(10^{-5}\right) \\
\end{array}$ & $3.7\left(10^{-5}\right)$ & $3.2\left(10^{-4}\right)$ & $4.3\left(10^{-5}\right)$ \\
\hline & {$[4]$} & $\begin{array}{l}\text { AWGN, BPSK, } \\
\mathrm{L}=32, \mathrm{CRC}=16\end{array}$ & ----- & ----- & ----- & $\mid----$ & ----- & ---- & $2.7\left(10^{-5}\right)$ & ----- \\
\hline & [4] & $\begin{array}{l}\text { AWGN, BPSK, } \\
\mathrm{L}=1024, \mathrm{CRC}=16\end{array}$ & ----- & ------ & ----- & $\mid----$ & ---- & ---- & $2.5\left(10^{-5}\right)$ & ----- \\
\hline \multirow{6}{*}{512} & {$[14]$} & $\begin{array}{l}\text { AWGN, QAM, } \\
\mathrm{L}=8, \mathrm{CRC}=8\end{array}$ & $2.3\left(10^{-4}\right)$ & $2.5\left(10^{-4}\right)$ & $3.2\left(10^{-4}\right)$ & ---- & ------ & $2.7\left(10^{-4}\right)$ & $2.9\left(10^{-4}\right)$ & $3.6\left(10^{-4}\right)$ \\
\hline & [14] & $\begin{array}{l}\text { Fading, QAM, } \\
\mathrm{L}=8, \mathrm{CRC}=8\end{array}$ & $3.4\left(10^{-4}\right)$ & $4.2\left(10^{-4}\right)$ & $5\left(5 \times 10^{-4}\right)$ & ------ & ----- & $4\left(10^{-4}\right)$ & $4.7\left(10^{-4}\right)$ & $5\left(10^{-2}\right)$ \\
\hline & {$[10,12]$} & $\begin{array}{l}\text { AWGN, BPSK, } \\
\mathrm{L}=8\end{array}$ & $0.5\left(10^{-5}\right)$ & $3.2\left(10^{-5}\right)$ & $5.2\left(10^{-5}\right)$ & $\begin{array}{l}6.8 \\
\left(10^{-5}\right)\end{array}$ & $\begin{array}{l}3.4 \\
\left(10^{-4}\right)\end{array}$ & $2.8\left(10^{-4}\right)$ & $3.7\left(10^{-5}\right)$ & $4.2\left(10^{-5}\right)$ \\
\hline & {$[12]$} & $\begin{array}{l}\text { AWGN,BPSK, } \\
\mathrm{L}=8, \mathrm{CRC}=8\end{array}$ & ----- & ----- & ----- & |---- & $\begin{array}{l}3.4 \\
\left(10^{-5}\right)\end{array}$ & $2.9\left(10^{-5}\right)$ & $3.3\left(10^{-5}\right)$ & $4.1\left(10^{-5}\right)$ \\
\hline & [4] & $\begin{array}{l}\text { AWGN, BPSK, } \\
\mathrm{L}=32, \mathrm{CRC}=16\end{array}$ & ---- & ----- & ------ & ----- & ------ & ------ & $2.4\left(10^{-5}\right)$ & ----- \\
\hline & [4] & $\begin{array}{l}\text { AWGN, BPSK, } \\
\mathrm{L}=1024, \mathrm{CRC}=16\end{array}$ & ----- & ----- & ----- & $\mid----$ & ----- & ---- & $2.1\left(10^{-5}\right)$ & ----- \\
\hline \multirow{3}{*}{1024} & [14] & $\begin{array}{l}\text { AWGN, QAM, } \\
\mathrm{L}=8, \mathrm{CRC}=8\end{array}$ & $\begin{array}{l}2 \\
\left(1.7 * 10^{-3}\right)\end{array}$ & $\begin{array}{l}2 \\
\left(1.2 * 10^{-3}\right)\end{array}$ & $2.9\left(10^{-4}\right)$ & ----- & ------ & $\begin{array}{l}2 \\
\left(7 * 10^{-3}\right)\end{array}$ & $2\left(5^{*} 10^{-3}\right)$ & $3.5\left(10^{-4}\right)$ \\
\hline & [14] & $\begin{array}{l}\text { Fading, } \mathrm{QAM}, \\
\mathrm{L}=8, \mathrm{CRC}=8\end{array}$ & $3.3\left(10^{-4}\right)$ & $4.2\left(10^{-4}\right)$ & $4\left(4 * 10^{-3}\right)$ & |---- & ----- & $4\left(10^{-4}\right)$ & $4.7\left(10^{-4}\right)$ & $4.6\left(10^{-2}\right)$ \\
\hline & [10] & $\begin{array}{l}\text { AWGN, BPSK } \\
\mathrm{I}=8\end{array}$ & $0.3\left(10^{-5}\right)$ & $2.5\left(10^{-5}\right)$ & $4.6\left(10^{-5}\right)$ & $\begin{array}{l}6.7 \\
\left(10^{-5}\right)\end{array}$ & ----- & ------ & ----- & ----- \\
\hline \multirow{2}{*}{2048} & [14] & $\begin{array}{l}\text { AWGN, QAM, } \\
\mathrm{L}=8, \mathrm{CRC}=8\end{array}$ & $2\left(3 * 10^{-3}\right)$ & $2.5\left(10^{-4}\right)$ & $2.7\left(10^{-4}\right)$ & ------ & ----- & $2\left(10^{-2}\right)$ & $2.7\left(10^{-4}\right)$ & $3\left(10^{-4}\right)$ \\
\hline & [10] & $\begin{array}{l}\text { AWGN, BPSK, } \\
\mathrm{L}=8\end{array}$ & $-0.3\left(10^{-5}\right)$ & $1.9\left(10^{-5}\right)$ & $4.2\left(10^{-5}\right)$ & $\begin{array}{l}6.6 \\
\left(10^{-5}\right)\end{array}$ & ----- & ---- & ---- & ----- \\
\hline
\end{tabular}

Table 4. Performance of polar code for different coding rates and environments

100 Journal of Electronic Systems Volume 8 Number 3 September 2018


coding rates. There is a slight advantage of LDPC over turbo code at high coding rate in most cases [5,15]. Also turbo code has an advantage of $1 \mathrm{~dB}$ over polar code with SCL $(L=8)$ especially at low coding rate at the cost of increased complexity [15]. On the other side polar code with SCL $(L=1024)$ and NB-LDPC achieved the best error performance with $0.5 \mathrm{~dB}$ advantage over turbo code at the cost of increased complexity by a factor of 80 [5]. The convolutional code with length of 512 showed a degrading performance compared to other codes [5,11].

For the case of 1024 coding length, the performance of LDPC and turbo codes are closed to each other with advantage in favor of turbo code at lower coding rates. Their performances are better than that of polar code with SCL $(\mathrm{L}=8)$ by about $1 \mathrm{~dB}$ over fading channel with higher complexity. Convolutional code showed the worst performance when the code length is $1024[11,15]$. At code length of 2048 or more almost similar behavior is produced, with the turbo code outperforms all other coding schemes at low error rates. For high coding rate, a better performance is provided by LDPC in most cases. Polar code with SCL $(\mathrm{L}=8)$ provided the least complexity with about $1 \mathrm{~dB}$ as a maximum loss at low coding rates. At code length of 4096 and 8192 , the performance of turbo code is the best at low coding rates with advantage of $0.5 \mathrm{~dB}$ over polar code with SCL (L=8) and LDPC. At high coding rates, turbo code and LDPC have the same performance. Again convolutional code showed the worst performance for all rates considered when the code length is equal or higher than $2048[11,15]$.

A comparison is made between turbo, LDPC, and polar codes in [1] where turbo and polar codes are considered as strong candidates for ultra-reliable low latency communication (URLLC) and massive machine type communication (mMTC) at block lengths lower than 1024, coding rate from $1 / 2$ to $2 / 3$ at targeted BLER of $10^{-4}$ to $10^{-5}$. Turbo code showed slight improvement in performance over polar code at lower rate. In addition LDPC code show two drawbacks; the first is related to performance degradation of more than $1 \mathrm{~dB}$ at low coding rate and short length, while the second is the increased complexity at low rate compared to other codes [1]. In term of complexity, turbo code showed an advantage over the other two codes at rate of $1 / 2$ with complexity increased linearly with data block length, unlike the cases of polar and LDPC codes.

Turbo and LDPC codes are combined in [21], where turbo code is considered for its flexibility and LDPC for its high throughput. Another combination is presented in [22], where the polar code is used for control channels while LDPC for data channel.

From above assessment it's clear that polar code with SCL $(L=8)$ decoding is the best choice at short coding length (64 and 128), while at higher lengths $(\geq 256)$, turbo code has the best error performance for low coding rates. LDPC has better error performance at high coding rates. The polar code with SCL $(L=8)$ decoding has the least complexity. Polar code with SCL (L=1024) and NBLDPC using GF(256) achieved good performance but their complexities are relatively high. Polar codes with SCL decoding $(L \geq$ 1024) give the best performance, while going for PSCL decoding reduces the code complexity. Further, convolutional code is not suitable to be used in $5 \mathrm{G}$ due to its poor performance. Considering other parameters related to channel coding implementation, polar code with SCL, Fast SSCL, and PSCL decoding algorithms required smaller area and lower power compared to LDPC codes [13]. NB-LDPC achieved better spectral efficiency compared to turbo code at coding rate of $1 / 2$ [6]. Further, turbo code has the advantage of providing flexibility and back-word compatibility over both LDPC and polar codes.

\section{Conclusion}

As a general conclusion one can say that there is no single channel coding candidate that can fulfil all $5 \mathrm{G}$ requirements. The study here reveals that polar code with Successive Cancelation List (SCL) decoding with small list length $(L=8)$ give the best choice at short lengths $(\leq 128)$ with relatively small complexity. Using larger list length $(L \geq 1024)$ in SCL decoding for polar code or using non-binary LDPC can achieve good performance on the expense of increased complexity with the advantage of better spectral efficiency. Partitioned Successive Cancellation List (PSCL) can be used to reduce complexity. Polar code with decoding algorithms based on SCL and PSCL requires smaller area and lower power consumptions when compared to LDPC codes. For higher coding lengths $(\geq 256)$ turbo code can provide better performance at low coding rates $(<1 / 2)$ while LDPC has better error performance at higher coding rates. Turbo code has the advantage of providing flexibility and back-word compatibility to $3 \mathrm{G}$ and $4 \mathrm{G}$ compared to LDPC and polar codes.

\section{References}

[1] Marsch, P., Bulakçý, Ö., Queseth, O., Boldi, M. (2018). 5G system design architectural and functional considerations and long term research, John Wiley \& Sons Ltd. 
[2] Gamage, H., Rajatheva, N., Latva-aho, M. (2017). Channel coding for enhanced mobile broadband communication in 5G systems," European Conference on Networks and Communications (EuCNC), Oulu, Finland, (June).

[3] M. ud in Mir., Kumar, S. (2015). Evolution of mobile wireless technology from 0G to 5G, International Journal of Computer Science and Information Technologies, 6 (3), (March).

[4] Mehta, H., Patel, D., Joshi, B., Modi, H. (2014). 0G to 5G mobile technology: a survey, Journal of Basic and Applied Engineering Research, 1 (6) (October).

[5] İpcan, O., Lentner, D., Xu, W. (2016). A comparison of channel coding schemes for 5G short message transmission, IEEE Globecom Workshops (GC Wkshps) Washington DC, USA, (December).

[6] Kim, H. (2015). Coding and modulation techniques for high spectral efficiency transmission in 5G and satcom, 23 ${ }^{\text {rd }}$ European Signal Processing Conference (EUSIPCO), Nice, France, (September).

[7] Maunder, R. (2016). The 5G channel code contenders, White Paper, AccelerComm Ltd., (August).

[8] 5G Forum, Republic of Korea, 5G vision, requirements, and enabling technologies, White Paper, (March 2016).

[9] Moreira, J., Farrell, P. (2006). Essential of error-control codes, Jhon Whiley \& Sons Ltd.

[10] Shannon, C. (1948). The mathematical theory of communication, Bell System Technical Journal, Vol. 27 (July).

[11] Tahir, B., Schwarzy, S., Rupp, M. (2017). BER comparison between convolutional, turbo, LDPC, and polar codes, $24^{\text {th }}$ International Conference on Telecommunications (ICT), Limassol, Cyprus, (May).

[12] MacKay, D., Neal, R. (1996). Near Shannon limit performance of low density parity check codes, Electronic Letters, 32 (18), (August).

[13] Ercan, F., Condo, C., Hashemi, S., Gross, W. (2017). On error-correction performance and implementation of polar code list decoders for 5G, 55 $5^{\text {th }}$ Annual Allerton Conference on Communication, Control, and Computing, Illinois, USA, (October).

[14] Arikan, E. (2009). Channel polarization: method for constructing capacity-achieving codes for symmetric binary-input memoryless channels, IEEE Transaction on Information Theory, 55 (7) (July).

[15] Gamage, H. (2017). Waveforms and channel coding for 5G, Master Thesis, University of Oulu, Oulu, Finland, (March).

[16] ETSI TR 138913 V14.2.0, 5G; Study on scenarios and requirements for next generation access technologies, May 2017.

[17] Brown, G. (2017). Serviced-based architecture for 5G core network, White Paper, Huawei Technology Co. Ltd, (November).

[18] Roh, W. (2014). 5G Mobile communication for 2020 and beyond, European Conference on Networks and Communications (EUCNC), Bologna, Italy, (June).

[19] Davey, M., Mackay, D. (1998). Low-density parity check codes over GF(q) IEEE Communication Letters, 2 (6) (June).

[20] 802.16-2012 - IEEE Standard for Air Interface for Broadband Wireless Access Systems,August 2012.

[21] Maunder, R. (2016). R1-1608584 Complementary turbo and LDPC codes for NR, motivated by a survey of over 100 ASICs, 3 GPP TSG RAN WG1 Meeting \#86bis, Portugal,10-14 (October).

[22] 3GPP TR 38.802, Study on new radio access technology physical layer aspects, V14.1.0, June 2017. 